\title{
El pozo San Enrique (Candín II) en Langreo. Análisis arquitectónico de los vestigios patrimoniales ligados a los espacios de producción y residencia
}

\author{
María Zapico López ${ }^{1}$ \\ Universidad de Nantes
}

RESUMEN

Uno de los símbolos del pasado industrial en el concejo de Langreo lo encarna el Pozo San Enrique, en La Felguera, clausurado en 2013. Los cotos hulleros en los que se radica, constituyen uno de los espacios de explotación de carbón de hulla de más larga tradición dentro municipio, adquiridos en el siglo XIX por Alejandro Aguado, Marqués de las Marismas, al objeto de extraer este mineral. La iniciativa de construir un pozo de extracción vertical, además de un grupo de viviendas para obreros, partió de la Sociedad Fábrica de Mieres, en la segunda década del siglo XX. Sin embargo, fue concluido casi dos décadas después por Minas de Langreo y Siero que se ocupó de su explotación, ininterrumpidamente, hasta que pasó a formar parte de HUNOSA. Como muestra de la labor productiva acontecida en este espacio encontramos numerosos vestigios arquitectónicos significativos del patrimonio industrial asociados a viviendas para obreros.

\section{PALABRAS CLAVE}

Pozo San Enrique, Langreo, arquitectura industrial, vivienda obrera, minería.

\section{ABSTRACT}

One of the symbols of the industrial past of the municipality of Langreo is the San Enrique (Candin II) Colliery, in la Felguera, recently closed, in 2013. This coal-mining area, in which the San Enrique Colliery is located, is one of the places with a longer coal-mining tradition within Langreo and it was acquired by Alejandro Aguado, Marquis of Marismas, to the coal mineral extraction in the nineteenth century. The initiative to build a vertical extraction coal shaft, and a group of workers' houses, came from the Sociedad Fábrica de Mieres, in the second decade of the twentieth century. However, it was concluded, nearly two decades after, by Minas de Langreo y Siero what uninterruptedly exploited it until it became part of HUNOSA. As a way to the productive work that took place in this point we can find numerous and significant architectonic remains of industrial heritage associated with workers' lodgings.

\section{KEYWORDS}

San Enrique Colliery, Langreo, industrial architecture, workers' housing, mining.

Beneficiaria del Programa “Clarín”- Marie Curie-Cofund de Ayudas Postdoctorales del Principado de Asturias en la Universidad de Nantes 
En el contexto de la Revolución Industrial, dentro del marco geográfico del Principado de Asturias, Langreo se erigió como uno de los enclaves en el que la industrialización se dejó sentir de modo más intenso, sobresaliendo por su riqueza carbonifera. En este sentido, los cotos hulleros radicados en las inmediaciones del río Candín, en La Felguera, cercanos a las vías de la estación de Vega del Ferrocarril de Langreo, constituyen uno de los centros de explotación más antiguos del concejo en los que empezó a extraerse carbón de hulla mediante la minería de montaña en el siglo XIX de la mano de Alejandro Aguado, Marqués de las Marismas. No fue hasta la segunda década del siglo $\mathrm{XX}$ cuando se inició la profundización de un pozo vertical, junto con un grupo de viviendas obreras, por parte de la Fábrica de Mieres. No obstante, la construcción quedó paralizada $\mathrm{y}$, quien verdaderamente acomete las obras del pozo e instalaciones auxiliares y lo explota, a partir de la década de 1930, fue la Sociedad Anónima Minas de Langreo y Siero, dentro del grupo Lláscaras.

La Sociedad Anónima de Minas de Langreo y Siero, con domicilio en Marqués de San Esteban, Gijón, fue constituida en $1925^{1}$. Sus orígenes los encontramos en la Sociedad de Minas de Carbón de Siero y Langreo, propiedad del andaluz Alejandro Aguado, Marqués de las Marismas, en ella se aunaban diversos cotos hulleros de ambos concejos asturianos. Tres años después de su fallecimiento, acaecido en 1842, fueron subastadas sus posesiones, yendo a parar los derechos de estas minas a Fernando Muñoz Sánchez, duque de Riánsares, quien tuvo un papel trascendental en el Ferrocarril de Langreo. A partir de 1853, tras una serie de gestiones, entra en escena Adolfo d'Eichthal y nace una nueva sociedad en la que se fusionaron sus minas con las explotaciones Riánsares, dando lugar a la Empresa Carbonera de Siero y Langreo $^{2}$.

\footnotetext{
Sus socios fundadores fueron cuatro, Pedro Pidal y Bernaldo de Quirós, Manuel Loring Martínez, Roque Pidal y Bernaldo de Quirós e Ignacio Fernández de la Lomera y Guzmán. En Anuario de Minería y Metalurgia de España, 1942 y DÍAZ GONZÁLEZ, María del Mar, Las acciones y obligaciones del archivo de HUNOSA. Composiciones formales y estética del trabajo (1833-1973), HUNOSA, Oviedo, 2007, p. 150.

2 En OJEDA, Germán, Asturias en la industrialización española 1833-1907, Siglo XXI de España Editores, Madrid, 1985, pp. 28 y 40.
}

Paralelamente, el 17 de septiembre de 1844, se constituyó en Londres la Asturian Mining Company, presidida por el financiero Gedeón Colguhoun, la cual fijó su emplazamiento en Mieres del Camino, donde la Sociedad adquirió minas de carbón. Pese a su corta vida, ya que la compañía se disolvió en 1849, junto a las minas de carbón, se montó una fábrica metalúrgica en las inmediaciones del río Caudal. No obstante, dejó tras de sí unas explotaciones industriales recién concebidas de las que terminó haciéndose cargo el grupo de Fernando Muñoz, desde el momento en que León Lillo y Juan Grimaldi (testaferros de Fernando) compraron la empresa. Poco después, tras un viaje de Riánsares al Principado de Asturias, se creó la gran sociedad Compagnie Miniere et Méttallurgique des Asturies, aunque sus planes se vieron truncados por la pérdida de influencia de Riánsares y Lillo. Es este el momento, 1857, en el que Juan Grimaldi y Riánsares vendieron estas instalaciones, radicadas en Mieres, al empresario francés Numa Guilhou. Poco después, en 1861, Guilhou se propuso acometer un proyecto empresarial más ambicioso, para ello adquirió al duque de Riánsares la Fábrica de Aceros de Lena y minas de carbón que este poseía en Langreo. De este modo, se conformó en sociedad la Houlliére et Metallurgique des Asturies, en la que Numa Guilhou, Berthier y Alfonso Marteville tenían como objetivo aglutinar diversas explotaciones mineras y establecimientos siderúrgicos.

Sin embargo, la Houlliére et Metallurgique des Asturies fracasó por problemas con la red de comunicaciones y, en 1868, se hizo cargo de la fábrica Numa Guilhou en solitario. En 1879, con el impulso técnico que proporcionó el ingeniero Jerónimo Ibrán, se creó la Fábrica de Mieres Sociedad Anónima que aglutinaba fábricas dedicadas al trabajo del acero y forja e integró como accionistas a los colaboradores de la antigua Houlliére et Metallurgique des Asturies, quedando las explotaciones mineras en un plano secundario ${ }^{3}$. Pese a todo, las minas de carbón que integraban el patrimonio de la Fábrica de Mieres se distribuían en varios puntos de la geografía asturiana: en el concejo de Mieres poseía los grupos Nicolasa, Baltasara, Mariana y Coruxas; en Pola de Lena, Bárzana y Muñón

En MUÑIZ SÁNCHEZ, Jorge, "Minas de Langreo y Siero" en URÍA, Jorge (Coord.): El Archivo Histórico de HUNOSA, HUNOSA, 2008, pp. 134-143. 


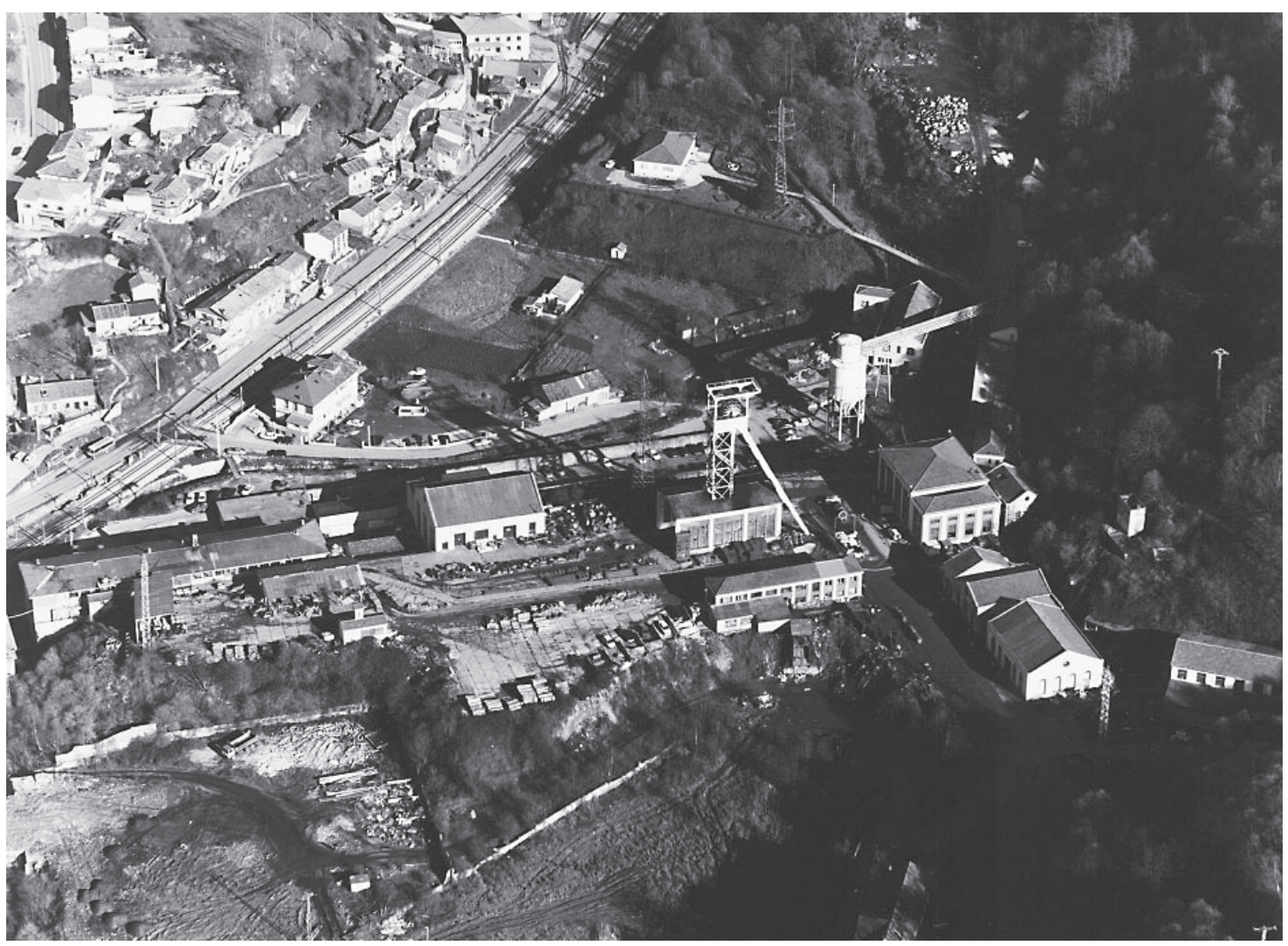

Fig. 1. Vista aérea del Pozo San Enrique (Candín II), La Felguera, año 2000. Fuente: Servicio de Patrimonio de HUNOSA

Cimero; en Oviedo (Tudela) Minas del Viso y Coto Tudela; en Langreo, Respinedo, Lláscaras y Sama; y en los concejos de Quirós, Teverga y Proaza el grupo Quirós.

A partir de 1925, parte de las explotaciones carboníferas fueron vendidas, separándose el negocio hullero del metalúrgico y surgiendo, finalmente, la Sociedad Minas de Langreo y Siero que a su vez agrupaba tres grupos: Lláscaras en La Felguera, Pumarabule, en Carbayín y el Viso en el Viso.

En la década de 1970, Minas de Langreo y Siero se integró en el patrimonio de la empresa estatal HUNOSA.

La principal explotación de Minas de Langreo y Siero, en Langreo, la constituye el Pozo San Enrique, más conocido en la actualidad como Candín II, y clausurado a comienzos de 2013, aunque en él se mantienen tareas de mantenimiento. Emplazado en la margen derecha del río Candín, circundan sus terrenos varias vías de comunicación: un ramal del antiguo Ferrocarril de Langreo y la carretera que discurre desde La Felguera a Tuilla.

Como señalábamos, los cotos hulleros denominados como grupo Lláscaras, en los que se radica este pozo propiedad de la Fábrica de Mieres Sociedad Anónima, son unos de los más antiguos explotados en Asturias.

Fue partir de 1918 cuando la sociedad Fábrica de Mieres decidió acometer las obras de perforación de un pozo de extracción vertical ${ }^{4}$ de 4,5 metros de diámetro útil para el aprovechamiento de las capas hulleras emplazadas por debajo del nivel de los valles. Sin embargo, la campaña constructiva fue interrumpida en el momento que se alcanzaron los 66 metros de profundidad, debido a las dificultades económicas por las que atravesaba la empresa en aquel entonces. Se trataba de un periodo complicado para la Fábrica de Mieres puesto que la empresa, como tantas otras de Asturias, se vio

\footnotetext{
4 La inauguración de las obras tuvo lugar el cinco de enero de 1918 cuando se llevó a cabo la bendición de esta profundización en presencia del cura párroco de Sama, Gerardo López y el capellán de la Fábrica de Mieres. Sería el padrino Enrique Pidal, hijo del Marqués de Villaviciosa. Posteriormente se ofrecería un banquete al que asistieron los Marqueses de Villaviciosa y sus hijos, el Conde de Mieres, señores de la Somera y Orueta, ingenieros de la Sociedad y jefes de movimiento de material, tracción, d vías y obras del Ferrocarril de Langreo. En Revista Industrial-Minera Asturiana, 16/02/1918.
} 
afectada por un ambiente de crisis generalizada una vez finalizada la Primera Guerra Mundial.

No fue hasta once años después, en 1929, cuando Minas de Langreo y Siero se propuso reanudar la profundización del pozo. En ese momento, la dirección de la empresa se encontró ante la disyuntiva de continuar las obras iniciadas por la Fábrica de Mieres, para finalizarlas, o construir un nuevo pozo de mayor sección, puesto que el diámetro del anterior se presentaba insuficiente. Finalmente, se decantaron por la primera opción para, de este modo, poder aprovechar las instalaciones ya ejecutadas ${ }^{5}$. Entre finales de 1931 y principios del año siguiente estaban prácticamente finalizadas las labores constructivas y se estaba trabajando en la estructura del castillete definitivo. Del mismo modo, se estaba llevando a cabo la instalación de la máquina de extracción, de los compresores de aire, de la sala de bombas y de otras dependencias ${ }^{6}$, previendo dar comienzo a la explotación del pozo en 1933.

En lo que se refiere a los inmuebles levantados, se ejecutaron en dos fases constructivas diversas. De un lado, estaban los edificios concebidos ex novo para servir al pozo de la década de 1930. De otro, aquellos que surgieron con posterioridad, en un momento en el que se expanden las instalaciones cuando o bien se amplían las edificaciones primigenias o bien se materializan otras de nueva planta.

Dentro de la primera fase constructiva, el edificio documentado más antiguo es el originariamente destinado a taller de ajuste $\mathrm{y}$ fragua $^{7}$, datado en 1936 según una copia de su proyecto fechada en junio de ese año ${ }^{8}$. De acuerdo con el plano, estaba integrado por dos espacios adosados, uno destinado a taller y el otro a fragua, unidos por medianera. El destinado a taller se organizaba a partir de

5 Las previsiones de la empresa eran que ante la situación estratégica de la mina, una zona rica en hulla, en primer lugar se llevaría a cabo este pozo y si la coyuntura era propicia se construiría un nuevo pozo de mayor extensión quedando el primero como auxiliar. En Estadística Minera y Metalúrgica de España, Madrid, 1932, pp. 324 y 325.

$6 \quad$ En Estadística Minera y Metalúrgica de España, Madrid, 1933, pp. 328-329.

7 Se encontraba ubicado de manera perpendicular al que hoy es el edificio de la antigua casa de aseo. Plano general del exterior del grupo Lláscaras, C/20.20, Fondo de Minas de Langreo y Siero. Archivo Histórico de HUNOSA Pozo Fondón.

$8 \quad$ Edificio para fragua, detalles y ampliaciones, Lláscaras. C/28.10, Fondo de Minas de Langreo y Siero. Archivo Histórico de HUNOSA Pozo Fondón. una planta rectangular, con mayor desarrollo; mientras, el reservado para fragua se resolvía a partir de un volumen cúbico. Esta compartimentación también se advertía al exterior, a través de la apertura de un mayor número de vanos para el área correspondiente al taller mecánico. Por su parte, los lienzos murales se animaban mediante la inserción de vanos con recercado y remate en arco de medio punto. Visualmente, estos quedaban unidos gracias a una moldura que arrancaba del salmer de cada uno hasta el del arco contiguo y a través de la utilización de un antepecho corrido. En lo que concierne a las fachadas laterales, incluían un mismo prototipo de vanos junto a un característico óculo en el hastial, que funcionaba como respiradero. Con posterioridad, en 1949, el taller experimentó un recrecido destinado a acoger un cuarto de soldadura y otro para aseos que, en lo básico, continuaba la impronta estilística del edificio primigenio. Con todo, los parámetros estilísticos que rigen esta construcción, sustentados en una composición mural basada en el equilibrio, la sobriedad y las líneas puras, nos permiten hablar de una impronta clasicista y coherencia estilística que contagia a las demás construcciones de la fase de la década de 1930. En este sentido, encontramos, además, cierta similitud con la composición de otras instalaciones mineras como el cercano Pozo Pumarabule, en Carbayín, concretamente su casa de máquinas, llevada a cabo por la Fábrica de Mieres en la segunda década del siglo XX. De otro lado, hallamos notables diferencias con el aspecto de los pozos asentados en el mismo concejo de Langreo y promovidos por otras empresas como la S.M.D.F. o Carbones de La Nueva.

De forma semejante al anterior se resuelve el conjunto actualmente inventariado como almacén ${ }^{9}$, el adyacente destinado almacén y sala de compresores ${ }^{10}$. En origen, en estos espacios se localizaban la sala de compresores, una central y las calderas. En su parte posterior, situada en una ladera al lado del espacio que ocupaba el plano inclinado, se ubica todavía la base de la chimenea de las calderas, derribada a finales de la década de 1980 . Se

\footnotetext{
9 Este edificio se encuentra incluido en el Inventario del Patrimonio Industrial Histórico de Langreo. Espacios de Trabajo, Ficha nº 128B, 2002, Ayuntamiento de Langreo. 10 Este edificio se encuentra incluido en el Inventario del Patrimonio Industrial Histórico de Langreo. Espacios de Trabajo, Ficha nº 129B, 2002, Ayuntamiento de Langreo.
} 


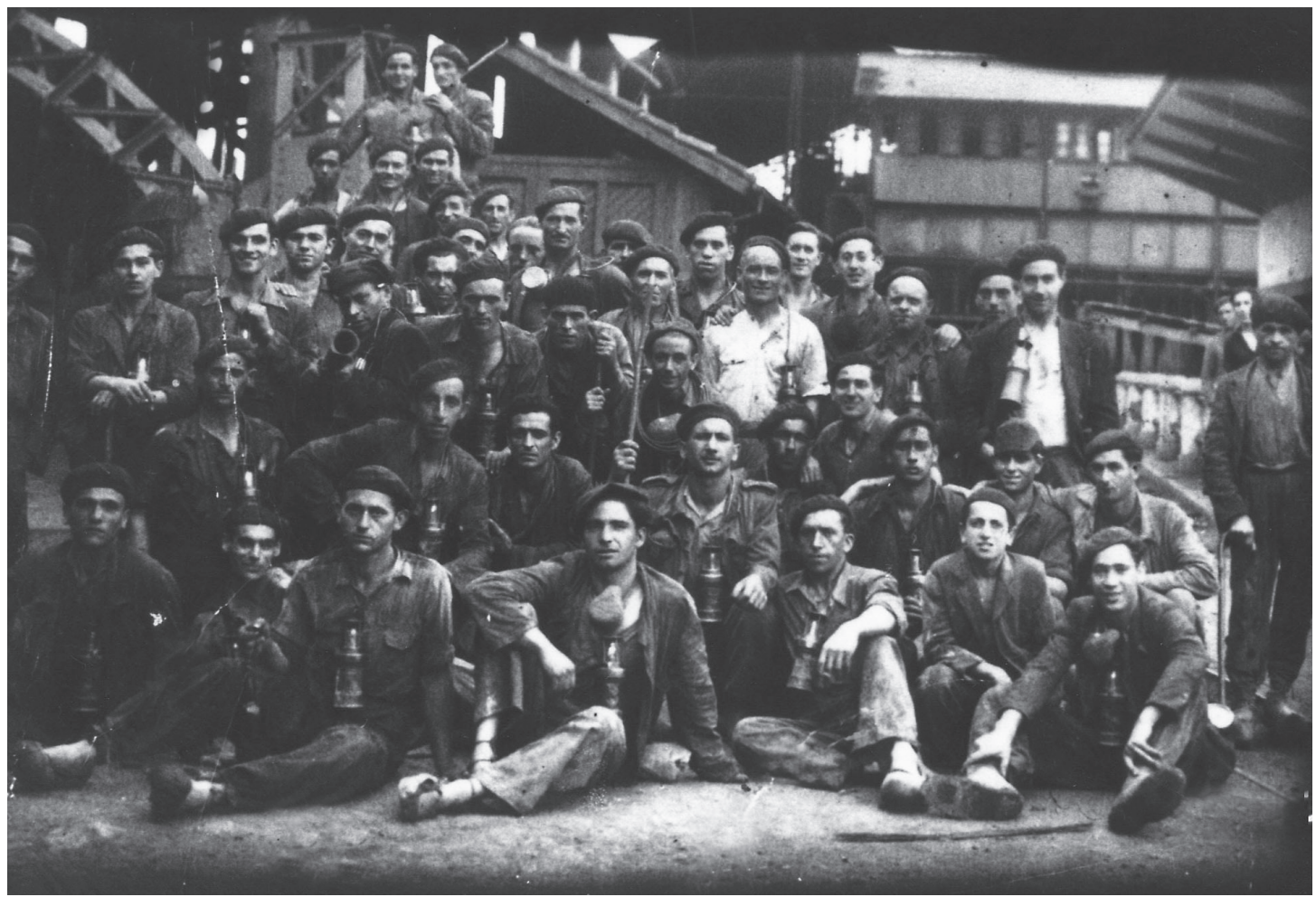

Fig. 2. Grupo minero del Pozo San Enrique, La Felguera, 1949. Fuente: Servicio de Patrimonio de HUNOSA

trata de un elemento de sección circular, ejecutado integramente en ladrillo, que funcionaba como arranque de una chimenea troncocónica. Entre sus principales atractivos encontramos una serie de molduras perpendiculares que aumentan su diámetro ascendentemente y motivos ornamentales, semejantes a dados, decorando su superficie.

De disposición paralela a la casa de máquinas, en su parte trasera, se encuentra una de las construcciones más singulares de la explotación: se trata del inmueble que albergaba el ventilador. Morfológicamente estamos ante un edificio sencillo, configurado a través de un volumen paralelepípedo al que se accede mediante una escalera realizada en piedra alojada en uno de sus laterales. Sus exteriores se articulan de modo semejante al del resto de edificios, a través de vanos alargados enlazados mediante molduras, incluyendo además respiraderos de sección circular en la zona de los hastiales.

Por último, dentro de la primera fase constructiva, mencionaremos el inmueble destinado a lavadero, alineado con la lampistería y próximo a la nueva casa de aseo el pozo. El inmueble parte de una base cuadrangular a la que se adosan diversos espacios; en lo que respecta a su alzado, se desarrolla mediante sótano y una sola altura. En su configuración exterior incorpora ventanales rectos ${ }^{11}$, de acusada verticalidad, abiertos con regularidad en sus paños murales. Asimismo, la cuidada integración de recercados y tratamiento de las impostas imprime cierta plasticidad al conjunto. De su exterior destaca, asimismo, la integración en el hastial de la fachada principal del emblema de la empresa así como la fecha de construcción, 1934. Desde el momento en el que entra en funcionamiento el nuevo lavadero del cercano Pozo Candín I, esta construcción pierde su función primigenia. Es así como en la actualidad su interior se presenta compartimentado para acoger un espacio dedicado a almacén y otro a taller eléctrico.

De otro lado, en el extremo superior derecho, cerrando la plaza, se emplaza el edificio para oficinas. Aunque de cronología algo posterior, 1942, estéticamente entronca con las construcciones referidas para la primera fase edificatoria del pozo. Documentalmente no podemos probar su autoría puesto que no se ha localizado su planimetría, aunque se suele atri-

\footnotetext{
1 En este caso no se utiliza el remate en arco de medio punto característico de esta primera fase constructiva y que comentábamos para los edificios anteriores.
} 


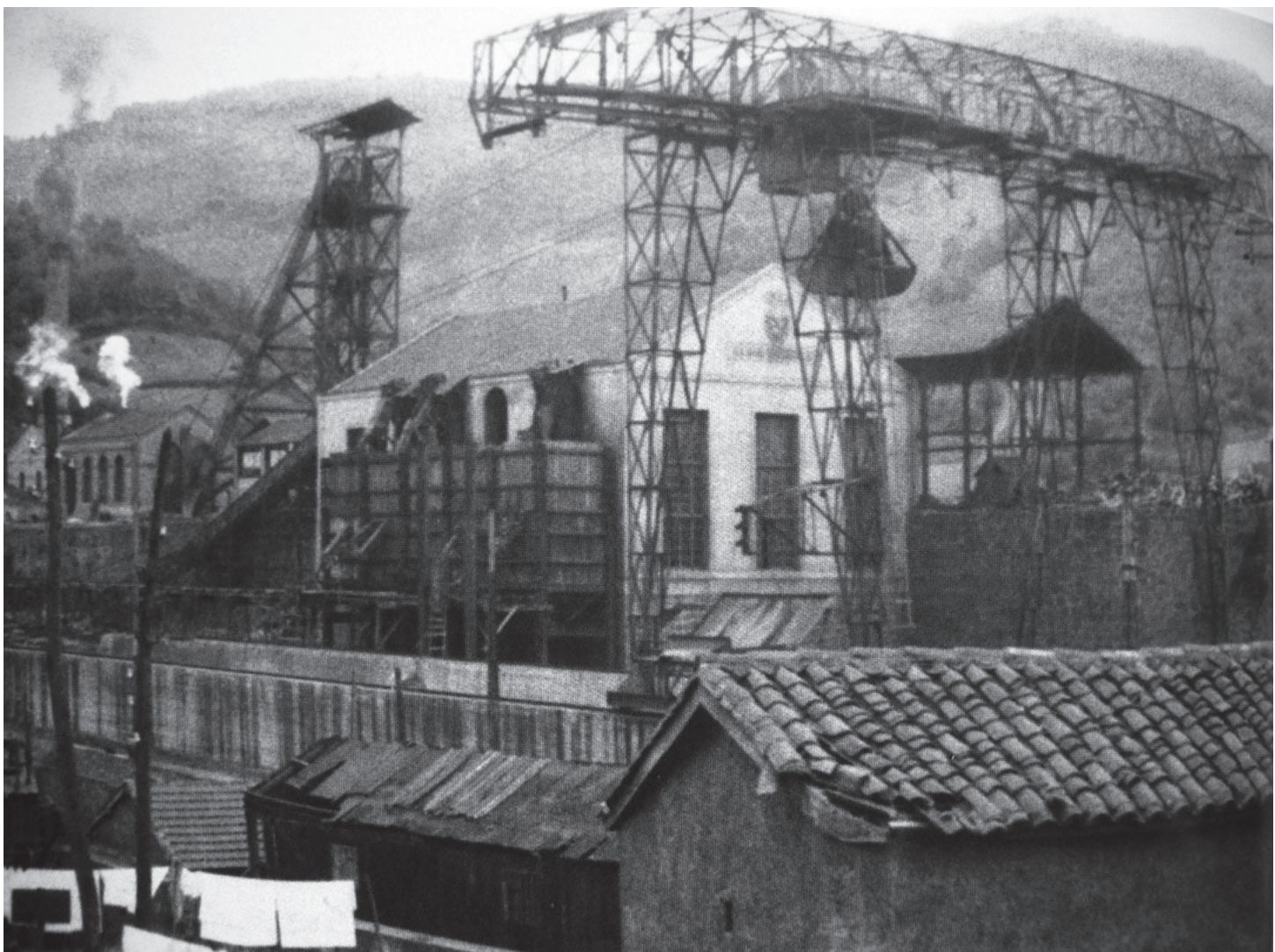

Fig. 3. Vista general del Pozo San Enrique, en primer término se sitúa el lavadero. Mediados del siglo XX. Fuente: Langreo en el pasado IV

buir a los hermanos Francisco y Federico Somolinos $^{12}$. El edificio presente es fruto de una serie de reformas ejecutadas a lo largo de los años. Su núcleo primigenio estaba integrado por dos alas de diversas dimensiones dispuesta de modo perpendicular, formando un L; con posterioridad se anexiona un volumen de una sola altura.

Especial interés reviste la tipología de ventanales elegidos para la planta superior, que combinan el remate en arco escarzano con el remate en medio punto y que, junto con el empleo de recercados y líneas de imposta, animan los cerramientos. Al igual que ocurriera en el edificio para antiguo lavadero, en el hastial de su fachada aloja el emblema de la empresa así como el año de su construcción.

Una nueva etapa constructiva da comienzo a partir finales de la década de 1950, con la ejecución de edificios en los que se adopta una nueva estética que difiere sustancialmente del clasicismo propio de las edificaciones anterio-

12 Catálogo Urbanístico del Concejo de Langreo y Catálogo Urbanistico del Plan Territorial especial de recuperación de los terrenos de HUNOSA en las Cuencas mineras. res. En primer lugar, hallamos la antigua casa de aseo para obreros ${ }^{13}$, en un punto donde primitivamente se ubicaban las oficinas del Grupo Lláscaras. Respecto a su cronología, se ha localizado una planimetría de su anteproyecto fechada en 1958. Aquí se representaba como una edificación de marcada horizontalidad, desarrollada en dos alturas. La articulación de sus muros se basaba en la incursión de pequeñas ventanas rectangulares, reservando para el piso bajo las ventanas triples. Por lo tanto, las fluctuaciones compositivas que se evidencian en el actual edificio podría ser fruto de una serie de variaciones incluidas en el proyecto final, no conservado, o debidas a reformas posteriores.

El edificio actual, de planta rectangular, presenta un exterior basado en la alternancia de muros y huecos gracias a la incursión de enormes ventanas rectas. Una de los puntos más destacables de su organización es la incorporación de volúmenes de diversa altura, así

13 Casa de Aseo para obreros, Lláscaras, C/23.19, Fondo de Minas de Langreo y Siero. Archivo Histórico de HUNOSA Pozo Fondón. 
como la ausencia de simetría en las vertientes de cubierta.

Adoptando una posición dominante en la plaza del pozo encontramos el conjunto que engloba los elementos centrales de la explotación, constituido por la casa de máquinas, castillete y embarque. Ejecutados en esta segunda etapa constructiva, aunque en la década de 1960, vinieron a sustituir a aquellos realizados para el primer pozo. Echando la vista atrás, el edificio originario de casa de máquinas ${ }^{14}$ presentaba una correlación estilística con la primera fase constructiva del pozo en cuanto a la configuración de sus vanos. Se trataba de una pequeña construcción de planta rectangular desarrollada en una sola altura y cubierta a dos aguas. Este inmueble fue sustituido por la actual casa de máquinas, uno de los elementos más emblemáticos de la explotación y destinado a albergar las máquinas de extracción ${ }^{15}$. Parte de una planta cuadrangular desarrollada por medio de sótano y una altura, compartimentada interiormente en dos espacios. Sus fachadas son tratadas mediante sucesiones de pilastras e impostas en ladrillo que contrastan con los paramentos revocados y pintados en verde, aligerados al calarse por vastos ventanales en vidrio. Su diseño racional, así como su nitidez, nos permiten incluir estilísticamente esta edificación dentro de los postulados imperantes en la época que entroncan con la lógica del movimiento moderno.

De otro lado, en lo que respecta al embarque se asemeja estéticamente al edificio de casa de máquinas. En este caso, se levanta a partir de estructura porticada hiperestática en hierro laminado. Su exterior aparece pautadamente reticulado a través de la incorporación de pilastras, impostas y vanos cuadrados, con la salvedad de las fachadas laterales que quedan al aire. Asimismo volvemos a encontrar la interesante tricromía de sus cerramientos mediante la utilización de ladrillo, vidrio y los muros revocados y pintados en verde ${ }^{16}$.

14 Véase la fotografía de la explotación primigenia contenida en http://vegalafelguera.wordpress.com/mineria/pozo-candin-ii-llascares-en-origen-llamado-pozo-san-enrique/.

15 Son los artilugios a través de los cuales se llevaba a cabo la tracción de las jaulas, por la caña del pozo, que mediante un movimiento rotatorio hacen que se vaya enrollando el cable del que se suspenden dichas jaulas.

16 De manera paralela al cobertizo, hacia el interior de la plaza, se ubica otra edificación que cumplía las funciones de oficinas para vigilantes y facultativos.
Por último, el primer castillete se estaba levantando desde 1931 y fue finalizado al año siguiente, aunque no se pondría en funcionamiento hasta $1933^{17}$. Este castillete ${ }^{18}$ primigenio estaba configurado a partir de un esqueleto metálico roblonado, de él apenas queda constancia documental ${ }^{19}$. Posiblemente coincidiendo con una reprofundización del pozo y la instalación de una nueva máquina de extracción, experimentó una serie de modificaciones en la década de 1960. Fue concebido, al igual que la estructura para embarque, por Ramón García Cánovas ${ }^{20}$. Morfológicamente el nuevo castillete se organiza a partir de un armazón triangulado metálico, con uniones mediante soldadura, que sustituían a los remaches de la primera época, sustentado sobre pilares o pies derechos arriostrados asentados sobre los extremos del brocal del pozo. En la parte superior hallamos las poleas por las que discurren los cables de los que penden las jaulas.

Imbuido en esta impronta racionalista se levantó también un edificio, no conservado, destinado a lampistería, situado paralelo al edificio de embarque, entre este y el de oficinas; realizado conforme a un proyecto de $1964^{21}$. Presentaba una planta rectangular de acusado sentido longitudinal compartimentada en varios espacios

17 En Estadística Minera y Metalúrgica de España, Madrid, 1933, pp. 328-329.

18 El castillete es una de las piezas más emblemáticas de la minería y con la que más se identifican este tipo de explotaciones de carbón de hulla. Funcionalmente se trata de un elemento que se ocupa de situar las poleas que aguantaban los cables de los que están suspendidas las jaulas en el ángulo y altura apropiados. A lo largo de la historia varias son las tipologías de castilletes que podemos hallar y numerosos los materiales en los que estos se construían, pero sin duda será el metálico el que predomine entre estos pozos del concejo de Langreo; desde que a partir de la Exposición Universal de París de 1867 irrumpiese el primer ejemplar de un castillete en hierro observaríamos una evolución en los mismos, que pasaría desde aquellos cuyas piezas se unían a través de roblones hasta aquellos que sustituyeron a estos por la soldadura. En SUÁREZ ANTUÑA, Faustino, Carbón para España. La organización de los espacios hulleros asturianos, KRK ediciones, Oviedo, 2006, p.229

19 Se conserva un fragmento de la estructura del castillete del pozo Lláscaras, aunque no del primitivo. Véase Castillete para el grupo Lláscaras, C/54.6, Fondo de Minas de Langreo y Siero. Archivo Histórico de HUNOSA Pozo Fondón.

20 En Catálogo Urbanístico del Plan Territorial especial de recuperación de los terrenos de HUNOSA en las Cuencas mineras, Ficha $\mathrm{n}^{\circ} 10$, Pozo Candín. Servicio de Patrimonio de HUNOSA.

${ }^{21}$ Proyecto de edificio para lampisteria, C/54.6, Fondo Minas de Langreo y Siero. Archivo Histórico de HUNOSA Pozo Fondón. 


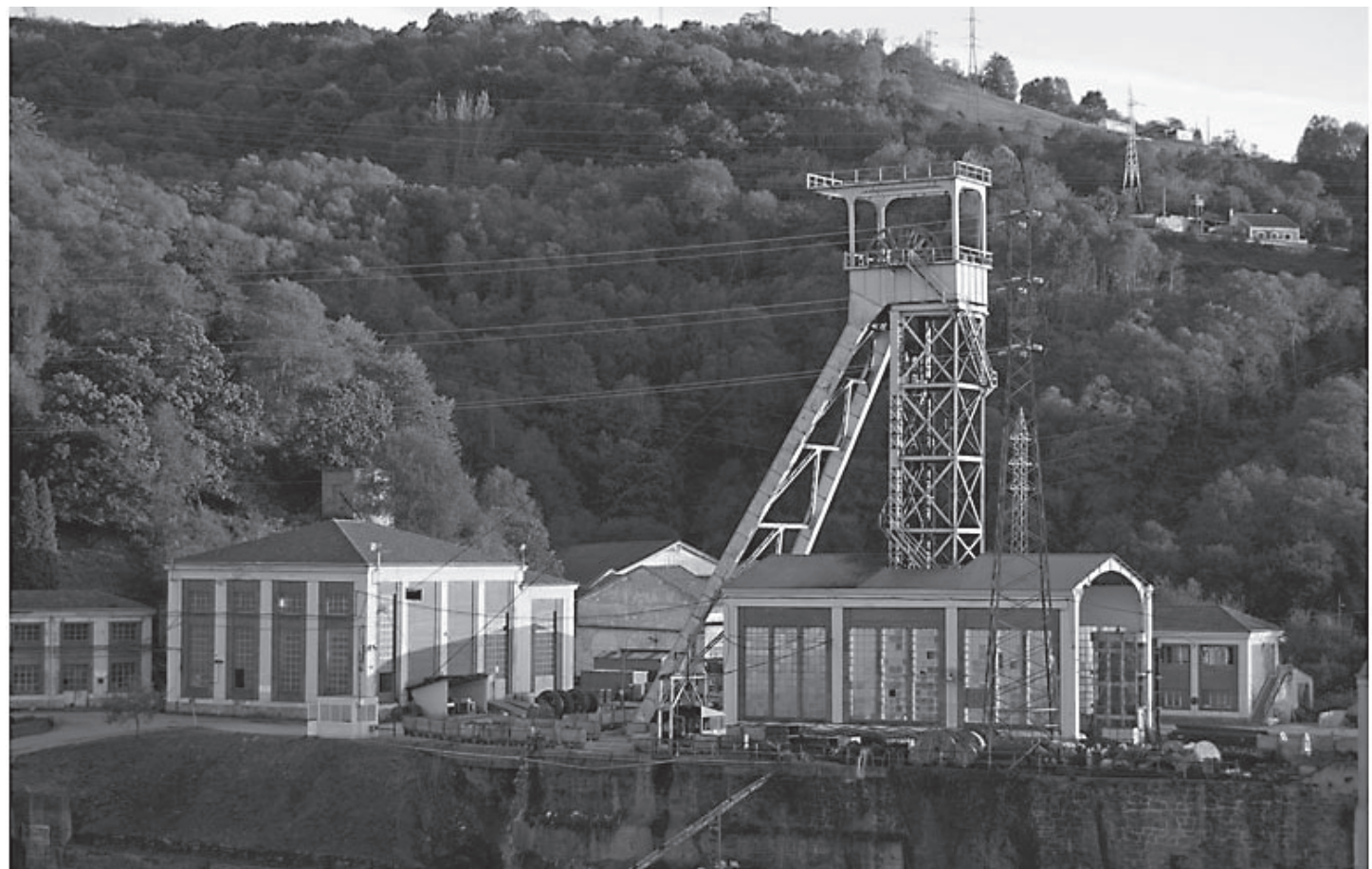

Fig. 4. Casa de máquinas, castillete y pabellón de embarque del Pozo San Enrique, estado actual. Fotografía de la autora

destinados a los bancos para lámparas eléctricas de cascos, para lámparas eléctricas de gancho o un armario perchero también para lámparas eléctricas de gancho. Sin embargo, con el paso del tiempo fue variando su compartimentación interior para adaptarse a las innovaciones técnicas producidas en este campo. Exteriormente nos topamos, de nuevo, con la incorporación en sus paños murales de una secuencia rítmica generada por la incorporación de pilares que acotan los vanos de la edificación presentes en la casa de máquinas y el embarque. En este sentido, también jugaba con texturas y variación de colores entre la plaqueta, vidrio y muro enfoscado. En lo que respecta a su cubierta, esta era disimétrica, con una parte a dos aguas sobre cerchas metálicas y otra a una sola vertiente para la caja de las escaleras.

En otro orden de cosas, el fenómeno que supuso la llegada de la Revolución Industrial no sólo tuvo incidencias en el plano productivo y en el patrimonio arquitectónico ligado al mismo, sino que también acarreó una fuerte convulsión a otros elementos constructivos tangibles, testimonio de la industrialización, como la vivienda para trabajadores.

En este sentido, y ligadas al pozo de extracción San Enrique o Candín II, situadas entre La Felguera y Tuilla, encontramos un número reducido de residencias para operarios adscritas a
Minas de Langreo y Siero. Se conocen con el nombre de Cuarteles de El Molinucu, localizados en un medio rural, en las inmediaciones del pozo San Enrique, entre el valle del Candín y Pajomal. Constituyen estas habitaciones la materialización de las políticas paternalistas de la empresa, que estimó oportuno erigir alojamientos para sus empleados con el fin de arraigar en ellos una serie de hábitos de docilidad que repercutirian en un mejor rendimiento en el trabajo.

Contamos con el plano del proyecto para estos cuarteles, el cual no contiene firma del arquitecto o encargado de realizarlo, únicamente podemos dilucidar a través de él la fecha de su construcción, 1918. Por lo tanto, parece evidente que este inmueble fue planteado conjuntamente con la perforación del Pozo San Enrique, cuando estos terrenos pertenecían todavía a la Fábrica de Mieres.

La composición elegida, en este caso, fue la de un edificio de planta rectangular, desarrollado en altura a través de dos plantas más el desván. Según el plano, el inmueble albergaría ocho viviendas, cada una con una orientación diversa. En lo que respecta a su distribución interior, inicialmente su planta baja poseía cocina, baño y un dormitorio; mientras, la planta superior, a la que se accedía mediante una escalera, estaba ocupada por piezas destinadas a dormitorios, separadas mediante un pasillo. 


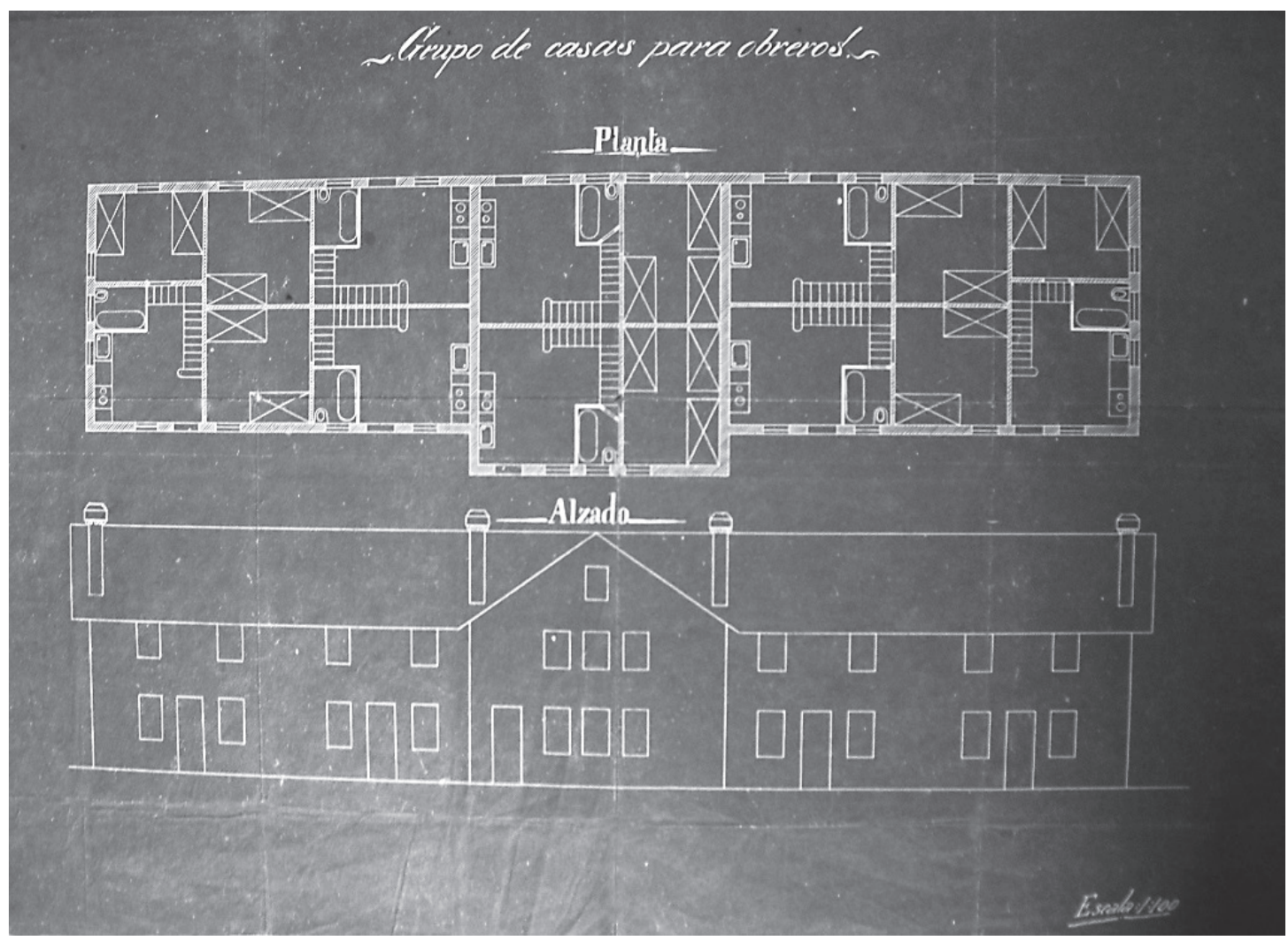

Fig. 5. Proyecto de los Cuarteles de El Molinucu, La Felguera, 1918. Fuente: Archivo Histórico HUNOSA Pozo Fondón

En el exterior del inmueble se observan los diferentes puntos de acceso a cada una de las viviendas y se distribuyen de la siguiente manera: la entrada a las dos casas dispuestas en los extremos se realizaba por sendas puertas abiertas en la fachada principal; de otro lado, las viviendas ubicadas en la parte central poseían su entrada unas por la parte anterior y otras por la cara posterior del edificio. Como remate se eligió una cubierta a doble vertiente, con el tejado del cuerpo central sobresaliendo de la línea de fachada y dispuesto de manera perpendicular a la techumbre del resto del edificio.

Si centramos ahora nuestra atención en la fachada principal ofrecida en el plano ${ }^{22}$ conservado, podemos percibir que esta se dividía en tres calles. La central, más adelantada, con tres filas de ventanas, las de las dos primeras plantas agrupadas en un conjunto de tres paralelas y con un solo hueco para el desván en la zona media, justo debajo del hastial de la cubierta (contiene además una puerta de acce-

22 Plano grupo de casas para obreros, 1918. C/ 27.21. Fondo de Minas de Langreo y Siero. Archivo Histórico de HUNOSA Pozo Fondón. so en el lateral izquierdo). Por su parte, cada uno de los cuerpos laterales de fachada cuenta con dos grupos de dos hileras de ventanas para cada piso, junto con las puertas de entrada en la zona inferior, reservando para la fachada posterior el resto de accesos a las viviendas. En lo que respecta a las fachadas laterales, la composición actual es resultado de las diferentes remodelaciones que ha experimentado a lo largo de la historia, lo que ha desembocado en un cambio evidente de su aspecto externo. Tal es el caso del pórtico edificado sobre pies derechos cubriendo toda la parte baja de la de la fachada principal del edificio, contrarrestando el desnivel entre el paramento de la parte central y los cuerpos laterales.

En cuanto a su factura, constructivamente se emplearon muros portantes ejecutados en ladrillo, mientras que la madera fue utilizada para la estructura entre pisos y el sostenimiento de la cubierta. Al exterior, las paredes van enfoscadas y pintadas; el tejado se realiza mediante teja plana.

Una vez elaborada esta aproximación descriptiva al inmueble, podemos concluir que se trata de un alojamiento colectivo que por su 
morfología se puede adscribir a la tipología de cuartel, aunque con la peculiaridad de contar con entradas individuales para cada vecino, de acuerdo con las teorías de salubridad y moralidad del momento. Estilísticamente, nos remite a modelos de colonias obreras centroeuropeos de carácter rústico.

A modo de conclusión, podemos establecer que el pozo San Enrique constituye uno de los ejemplos más singulares de la arquitectura industrial en Asturias por diversos aspectos. En primer lugar, por la larga tradición productiva de sus cotos hulleros, en las inmediaciones del río Candín (Langreo), que comenzaron a ser explotados en el siglo XIX por Alejandro Aguado. En segundo lugar, por erigirse como exponente de la evolución de este espacio productivo desde la minería de montaña, en su inicios, hasta que se emprendió la profundización de un pozo vertical en las primeras décadas del siglo XX, abriendo nuevos horizontes productivos mediante el aprovechamiento de las capas hulleras emplazadas por debajo del nivel del valle.

En lo que se refiere a los ejemplos arquitectónicos levantados en el espacio de trabajo, el análisis constructivo del Pozo San Enrique, a través de sus dos etapas constructivas bien diferenciadas, nos permite acercarnos a los numerosos vestigios industriales que conformaban sus instalaciones. Quedan así de manifiesto las interesantes propuestas en él empleadas, sobre todo en su primera etapa, en la que los proyectistas se decantaron por un vocabulario que entronca con los valores formales del clasicismo, con arcos de medio punto, respiraderos de sección circular, frontones o molduras que do- tan de plasticidad a los paramentos y unen visualmente los huecos abiertos rítmicamente en las fachadas. Todo esto contrasta con los recursos utilizados en la segunda etapa constructiva imbuida en la corriente racionalista, resaltando la nitidez y elementalidad constructiva. Tal es así que podemos concluir que, en su conjunto, en los inmuebles estudiados distinguimos esencialmente el uso reiterado planta rectangular con un desarrollo configurado por sótano y una sola planta. Por su parte, el resultado en alzado, es el de bloques paralelepípedos en cuyos lienzos murales se abren líneas de ventanas, siempre iguales, repetitivas, generando un ritmo alternante de macizos y huecos. Suelen ser vanos de dirección vertical y amplias dimensiones, lo cual proporcionaba magníficas condiciones lumínicas en sus interiores. Del mismo modo, los modelos de cubierta analizados son bastante repetitivos, casi siempre a doble o varias vertientes, asentadas sobre armaduras triangulares metálicas.

Por último, unido al espacio productivo hallamos un bloque residencial que proporcionaba alojamiento para los obreros a fin de obtener una mayor productividad, entroncando con los postulados del paternalismo industrial. En este caso, la arquitectura no guarda ningún paralelismo con aquella levantada para la extracción de carbón en el Pozo San Enrique, sino que se decanta por modelos habitacionales de tradición centroeuropea, sin que exista una unidad de lenguaje arquitectónico y sin que se pueda hablar de un estilo uniforme en el pozo y el espacio de residencia que sirviese como metáfora de la identidad corporativa. 